\title{
Octopaminergic modulation of contrast gain adaptation in fly visual motion-sensitive neurons
}

\author{
Diana Rien, Roland Kern and Rafael Kurtz \\ Department of Neurobiology, Faculty of Biology, Bielefeld University, PO Box 1001 31, 33501 Bielefeld, Germany
}

Keywords: behavioural state, Calliphora vicina, contrast sensitivity, neuronal adaptation, optic flow

\begin{abstract}
Locomotor activity like walking or flying has recently been shown to alter visual processing in several species. In insects, the neuromodulator octopamine is thought to play an important role in mediating state changes during locomotion of the animal [K.D. Longden \& H.G. Krapp (2009) J. Neurophysiol., 102, 3606-3618; (2010) Front. Syst. Neurosci., 4, 153; S.N. Jung et al. (2011) J. Neurosci., 31, 9231-9237]. Here, we used the octopamine agonist chlordimeform (CDM) to mimic effects of behavioural state changes on visual motion processing. We recorded from identified motion-sensitive visual interneurons in the lobula plate of the blowfly Calliphora vicina. In these neurons, which are thought to be involved in visual guidance of locomotion, motion adaptation leads to a prominent attenuation of contrast sensitivity. Following CDM application, the neurons maintained high contrast sensitivity in the adapted state. This modulation of contrast gain adaptation was independent of the activity of the recorded neurons, because it was also present after stimulation with visual motion that did not result in deviations from the neurons' resting activity. We conclude that CDM affects presynaptic inputs of the recorded neurons. Accordingly, the effect of CDM was weak when adapting and test stimuli were presented in different parts of the receptive field, stimulating separate populations of local presynaptic neurons. In the peripheral visual system adaptation depends on the temporal frequency of the stimulus pattern and is therefore related to pattern velocity. Contrast gain adaptation could therefore be the basis for a shift in the velocity tuning that was previously suggested to contribute to state-dependent processing of visual motion information in the lobula plate interneurons.
\end{abstract}

\section{Introduction}

Across different phyla (flies: Rosner et al., 2009, 2010; Chiappe et al., 2010; Maimon et al., 2010; Jung et al., 2011; locusts: Rind et al., 2008; birds: McArthur \& Dickman, 2011; rodents: Niell \& Stryker, 2010) processing of visual information was recently demonstrated to depend on the locomotor state of the animal (for review, see Maimon, 2011). Similar to norepinephrine in the vertebrate nervous system, the invertebrate neuromodulator octopamine plays a prominent role in the regulation of excitability (for review, see Roeder, 2005). In locusts, the biogenic amine octopamine is released during flight (Goosey \& Candy, 1980) and, in locusts as well as in other insects, has been shown to affect metabolism (Wegener, 1996) and the processing of visual information (Bacon et al., 1995; Erber \& Kloppenburg, 1995; Rind et al., 2008; Stern, 2009). In visual motion-sensitive neurons in the third visual neuropil of the fly (lobula plate tangential cells, LPTCs; for reviews, see Egelhaaf et al., 2002; Borst et al., 2010), flight activity as well as walking were shown to increase spontaneous and stimulus-induced activity (Chiappe et al., 2010; Maimon et al., 2010). Similar changes in neuronal response properties were elicited by application of the octopamine agonist chlordimeform (CDM) to the haemolymph (Longden \& Krapp, 2009, 2010; Jung et al., 2011). In the present study, we used CDM to induce a high-activity state in the

Correspondence: Dr R. Kurtz, as above.

E-mail: rafael.kurtz@uni-bielefeld.de

Received 29 February 2012, revised 6 June 2012, accepted 10 June 2012 nervous system as observed during the animals' locomotion. We applied specific stimulation paradigms that allowed us to explore the cellular basis of the state-dependent activity regulation of two individually identified LPTCs, the H1 neurons and the V1 neurons. Both neurons can be individually identified in the fly brain, and are thought to take over specific functions in the context of optic flow processing (Horstmann et al., 2000; Karmeier et al., 2003; Hennig et al., 2011). Whereas the $\mathrm{H} 1$ neuron is sensitive to horizontal motion (Eckert, 1980) and is assumed to receive direct retinotopic input, the V1 neuron gets input from other LPTCs and responds strongly to vertical motion (Krapp et al., 2001; Kurtz et al., 2001).

When presented with continuous motion at a fixed velocity, fly LPTCs respond with a rapid increase of membrane potential or spike rate to a transient peak, followed by a gradual decline to a tonic response level (Eckert, 1980; Hausen, 1982; Fig. 1A and E). These dynamic response characteristics are due to the properties of elementary motion detectors (Egelhaaf \& Borst, 1989), which provide the input to LPTCs, and to neuronal adaptation (for review, see Kurtz, 2011). The dynamic response characteristics of the H1 neuron and of another LPTC, the H2 neuron, were shown to be affected by CDM (Longden \& Krapp, 2010; Jung et al., 2011). In our study, we systematically tested how CDM affects motion adaptation. Adaptation of LPTCs was shown to result from cell-intrinsic changes as well as changes localized in presynaptic processing layers (Harris et al., 2000; Kurtz, 2007; Nordström \& O'Carroll, 2009). Our analysis of how $\mathrm{CDM}$ affects adaptation aims to clarify the cellular mechanisms 
A

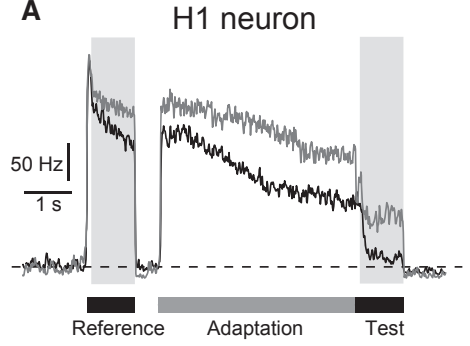

E

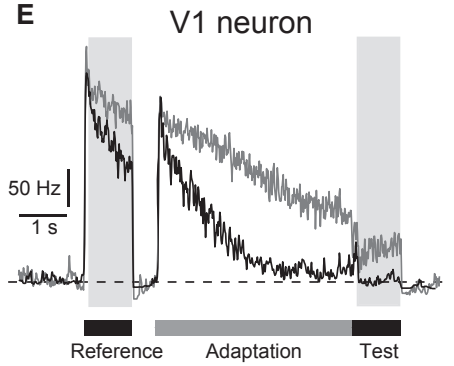

B

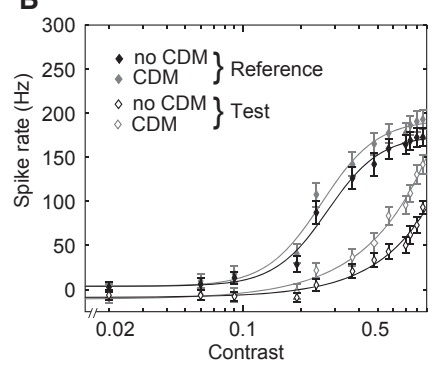

F

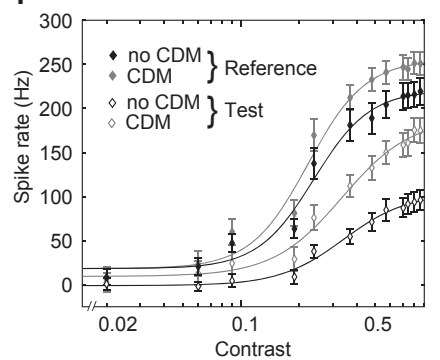

C

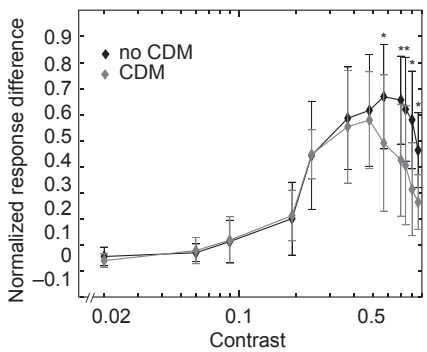

G

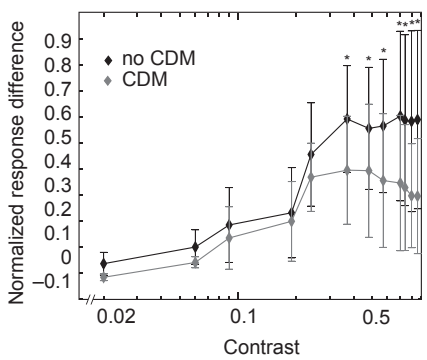

D i
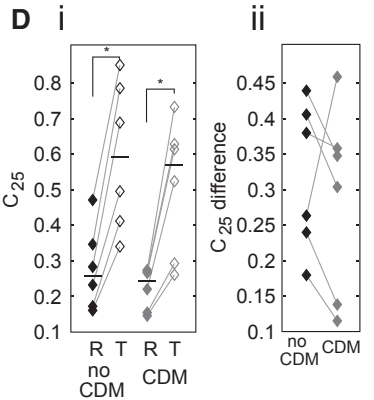

H i

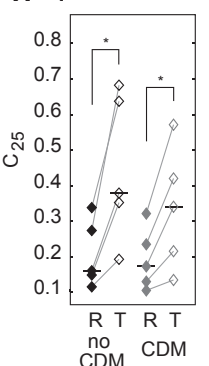

ii

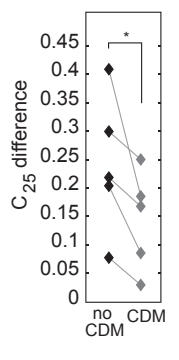

FIG. 1. Effect of chlordimeform (CDM) on contrast-response functions before and after motion adaptation. (A) Representative response of a H1 neuron to preferred-direction motion of a sinusoidal grating presented with a Michelson contrast of 0.7 during the reference and test intervals, and 0.95 during adaptation. The black and grey lines represent the responses before and after CDM application, respectively. The resting activity was subtracted from the responses. (B) Mean responses of $\mathrm{H} 1$ neurons before (filled symbols) and after adaptation (open symbols), each before (black) and after (grey) CDM application. Responses were calculated as the spike rate averaged in a 900 -ms time window starting $100 \mathrm{~ms}$ after stimulus onset, as indicated by the shaded areas in (A). Sinusoidal curves were fitted to the mean values (see Materials and methods for details). (C) Normalized differences between the unadapted and adapted responses before (black) and after (grey) CDM application. (D) (i) Contrast that evokes $25 \%\left(C_{25}\right)$ of the respective maximum response, as determined from the unadapted responses before CDM application. Each symbol represents data of one fly obtained before (R, reference; filled symbols) and after adaptation (T, test; open symbols), before (black) and after (grey) CDM application. The black horizontal bars represent the medians across all flies $(N=6)$. (ii) Differences in the $C_{25}$ before (black) and after (grey) CDM application. $(\mathrm{E}-\mathrm{H})$ Data for $\mathrm{V} 1$ neurons $(N=5)$, plotted in the same way as for $\mathrm{H} 1$ neurons. Asterisks represent statistical significance at the $5 \%$ significance level (paired $t$-test).

underlying the modulation of neuronal activity by CDM and to pinpoint its site of action within the visual system.

\section{Materials and methods}

\section{Animal preparation and electrophysiology}

Female blowflies (Calliphora vicina, 3-5 days old), taken from our laboratory stock, were briefly anaesthetized with $\mathrm{CO}_{2}$ and fixed to a small glass plate at the dorsal thorax. The legs were removed and the wounds were sealed with bee's wax. The wings and abdomen were immobilized with bee's wax. The proboscis was stretched out and waxed to the thorax. The head capsule was opened from behind, and the air sacs and trachea were removed. The orientation of the fly's head was aligned according to the symmetrical deep pseudopupil in the frontal region of both eyes (Franceschini, 1975).

The spike activity of a V1 neuron or a $\mathrm{H} 1$ neuron was recorded extracellularly in its respective output arborization in the right brain hemisphere using borosilicate glass electrodes (GC150TF-10; Clark Electromedical, Edenbridge, UK) with an outer diameter of $1.5 \mathrm{~mm}$. The electrodes were pulled with a Brown-Flaming electrode puller (P97; Sutter Instruments, San Rafael, CA, USA) and filled with $1 \mathrm{M}$ $\mathrm{KCl}$ resulting in a resistance of $1-5 \mathrm{M} \Omega$. The $\mathrm{H} 1$ neuron is unambiguously identifiable by its sensitivity to horizontal back-tofront motion in the frontal to frontolateral part of the visual field contralateral to the recording site, whereas the V1 neuron was identified by its sensitivity to downward motion in a similar but less extended part of the visual field (Hausen, 1984; Kurtz et al., 2001;
Krapp et al., 2001). Data were collected only from neurons that displayed sufficiently large extracellular spike amplitudes relative to the amplitude distribution of background noise, resulting in signal-to-noise ratios of at least $2.5: 1$. Spikes were detected by adjusting a voltage threshold. The resulting pulses were sampled at $5 \mathrm{kHz}$ and analoguedigital converted (DT 3001; Data Translation, Marlboro, MA, USA).

\section{Pharmacology}

To mimic the effects of octopamine, we applied the tissue-permeable octopamine receptor agonist CDM-HCl (CDM; Sigma-Aldrich, Dorset, UK; Evans \& Gee, 1980; Hollingworth \& Murdock, 1980). CDM was dissolved in water (aqua bidest.) as a $0.26 \mathrm{~mm}$ stock solution and stored at $-18{ }^{\circ} \mathrm{C}$. Before each experiment, the CDM stock solution was diluted in fly Ringer solution (see Kurtz et al., 2001 for composition) to obtain a final concentration of $2.6 \mu \mathrm{M}$. A drop of $10 \mu \mathrm{L} \mathrm{CDM}$ solution was applied to the fly's brain. This procedure was chosen for the following reasons: first, using the same procedure Longden \& Krapp $(2009,2010)$ reported robust increases in spontaneous and stimulus-induced activity in the neurons H1, V1 and V2 in Calliphora vicina, whereas similar but much weaker effects were obtained when a concentration of $0.26 \mu \mathrm{M}$ was used. Second, relating the amount of CDM to the body mass, Longden \& Krapp (2009) estimated the concentration of $2.6 \mu \mathrm{M}$ to be at the lower end of physiologically relevant concentrations of CDM. Effects of CDM on receptors for neuromodulators other than octopamine, which are known to occur at high concentrations of CDM (Costa et al., 1988; 
Hiripi et al., 1999), are thus kept as low as possible. Third, use of a higher concentration of CDM supplied to the haemolymph with a lower amount of solution ( $2 \mu \mathrm{L}$ containing $20 \mu \mathrm{M}$ CDM was used in Jung et al., 2011) was disfavoured, because the effective concentration of CDM was expected to depend more strongly on the amount of haemolymph present in the head capsule before application. Nonetheless, our procedure will lead to large variability of effective concentrations across different neuropils and across animals, given inevitable differences in brain sheath permeation and amount of haemolymph. Nevertheless, we regard this procedure as the best approach available so far to induce the neural activity level present during fictive locomotion in immobilized flies. In this context we emphasize that consistent results were obtained using application of CDM solution to the haemolymph (Longden \& Krapp, 2009, 2010; Jung et al., 2011), and that in the present study the major effects of $\mathrm{CDM}$ can be demonstrated even without normalization of data from individual flies.

\section{Visual stimulation}

Drifting sine-wave gratings were generated by a PC-controlled image synthesizer (Picasso, Innisfree, Cambridge, MA, USA) and displayed on a cathode ray tube (Tektronix 608, Wilsonville, OR, USA) at a frame rate of $183 \mathrm{~Hz}$. The monitor was centred at an azimuth/elevation $30 \% 0^{\circ}$ with an azimuth/elevation of $0 \% 0^{\circ}$ corresponding to the animal's frontal midline/equatorial plane, respectively. The monitor screen covered $82^{\circ} \times 85^{\circ}$ (horizontal $\times$ vertical extent) within the visual field of the fly.

\section{Adaptation protocols}

A green screen at mean luminance $\left(8.8 \mathrm{~cd} / \mathrm{m}^{2}\right)$ was shown for at least $5 \mathrm{~s}$ between individual trials to avoid accumulation of adaptation effects.

\section{Contrast-response protocol}

We modified the protocol from Harris et al. (2000) to determine the effect CDM has on motion adaptation of $\mathrm{H} 1$ and V1 neurons by measuring the neurons' contrast-response curves. The stimulus protocol consisted of a drifting sinusoidal grating presented in a reference-adaptation-test sequence, in which the reference and test stimuli were identical. The drifting grating was always shown on the entire screen and had a temporal frequency of $8 \mathrm{~Hz}$. Various contrasts were used for the reference/test stimuli to assess the neuron's contrast sensitivity function. The protocol started with the reference stimulus moving in the preferred direction for $1 \mathrm{~s}$, followed by a switch of the screen to mean luminance for $500 \mathrm{~ms}$. Afterwards, a high-contrast adapting stimulus (Michelson contrast 0.95) was presented for $4 \mathrm{~s}$, immediately followed by the test stimulus. The reference/test contrasts could assume values between 0.02 and 0.95 Michelson contrast (Figs 1 and 2). In the first set of experiments the adapting stimuli were moving in the neuron's preferred direction (Fig. 1). In the second set the adapting stimuli were moving in a direction that was approximately orthogonal to the neuron's preferred direction (Fig. 2). In pre-tests we adjusted the direction of the orthogonal stimulus so that the activity of the cells stayed as close to resting level as possible. This adjustment resulted in adapting stimuli moving at $115^{\circ}$ for $\mathrm{H} 1$, and at $10^{\circ}$ for $\mathrm{V} 1$, with $90^{\circ}$ and $0^{\circ}$ corresponding to downward and
A

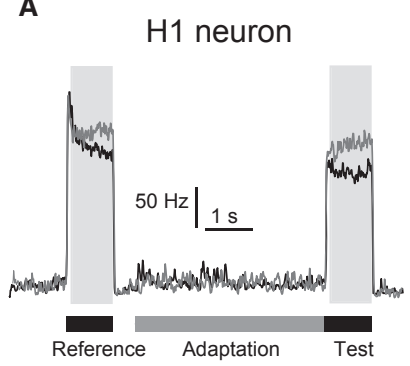

E

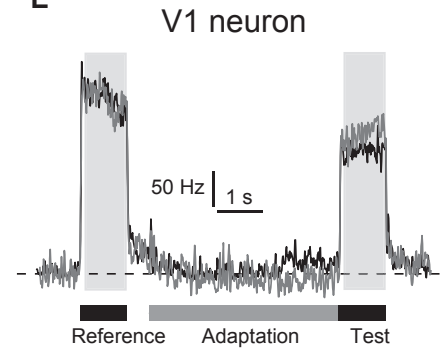

B

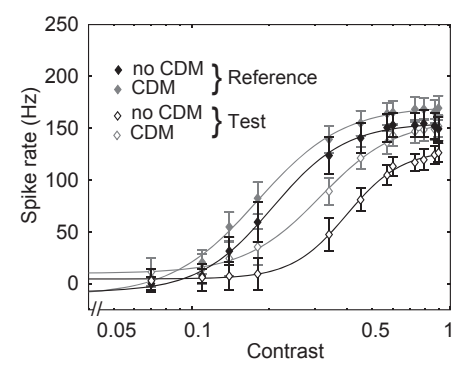

$\mathbf{F}$

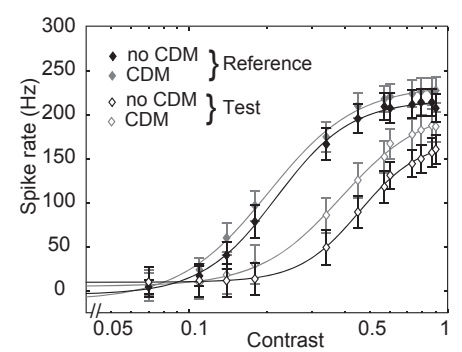

C

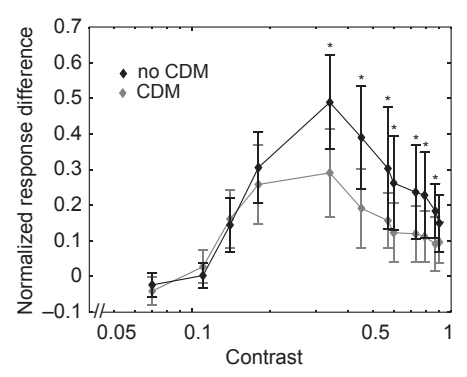

G

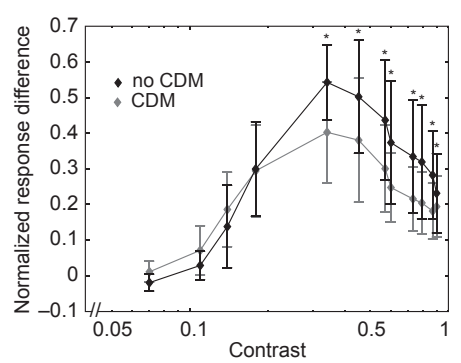

D i
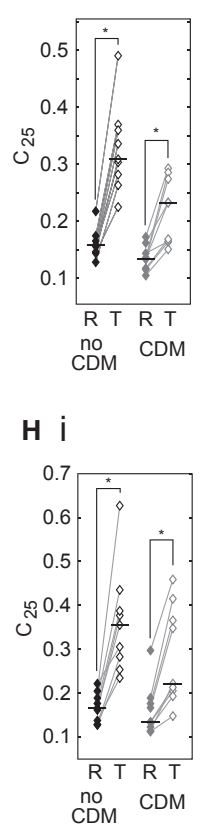

ii

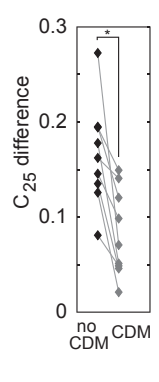

ii

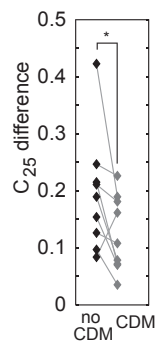

FIG. 2. Effect of chlordimeform (CDM) on contrast-response functions before and after adaptation with motion orthogonal to the preferred direction. (A) Representative response of a $\mathrm{H} 1$ neuron in a similar stimulus protocol as described in Fig. 1, but with downward motion of the grating during the adapting period, i.e. orthogonal to the preferred direction. The Michelson contrast was 0.73 for the reference/test stimuli and 0.95 for the adapting stimulus. The black and grey lines represent the responses before and after CDM application, respectively. (B) Mean responses of H1 neurons before (filled symbols) and after adaptation (open symbols), each before (black) and after (grey) CDM application. Responses were calculated as the spike rate averaged in a 900-ms time window starting 100 ms after stimulus onset, as indicated by the shaded areas in (A). (C) Normalized differences between the unadapted and adapted responses before (black) and after (grey) CDM application. (D) (i) Contrast that evokes 25\% $\left(C_{25}\right)$ of the respective maximum response, as determined from the unadapted responses before CDM application. Each symbol represents data of one fly obtained before (R, reference; filled symbols) and after adaptation ( $T$, test; open symbols), before (black) and after (grey) CDM application. The black horizontal bars represent the medians across all flies $(N=9)$. (ii) Differences in the $C_{25}$ before (black) and after (grey) CDM application. $(\mathrm{E}-\mathrm{H})$ Data for V1 neurons $(N=9)$, plotted in the same way as for H1 neurons. Asterisks represent statistical significance at the $5 \%$ significance level (paired $t$-test). 
back-to-front motion, respectively. For simplicity, the direction determined in this way is called 'orthogonal' in the following text. We obtained the following values: $\mathrm{H} 1$ resting activity: before CDM $26 \pm 8.7 \mathrm{~Hz}$, after CDM $34 \pm 7.7 \mathrm{~Hz}$; activity induced by orthogonal motion: before CDM $23 \pm 9.8 \mathrm{~Hz}$, after $\mathrm{CDM} 31 \pm 15.4 \mathrm{~Hz} ; N=9$; V1 resting activity: before CDM $27 \pm 7.7 \mathrm{~Hz}$, after CDM $45 \pm 14.1 \mathrm{~Hz}$; activity induced by orthogonal motion: before CDM $16 \pm 7.0 \mathrm{~Hz}$, after CDM $34 \pm 13.1 \mathrm{~Hz} ; N=9$.

We interpolated the data by fitting a sigmoid function

$$
f(x)=\frac{a * x^{b}}{x^{b}+c^{b}}+d
$$

In this equation the parameters $a$ and $b$ correspond to the saturation level and the slope of the function, respectively. The parameter $c$ controls the contrast gain reduction (shift along the $x$-axis), whereas $d$ controls the offset (shift along the $y$-axis). To determine the $C_{25}$ value the contrast that elicits $25 \%$ of the maximum response was taken from the fitted curve.

\section{Local vs. global adaptation}

To distinguish between local and global effects of CDM, the screen was divided into three parts. The protocol consisted of a reference stimulus, followed by an adaptation stimulus and a final test stimulus, which was identical to the reference. The reference/test grating was in every trial positioned at the centre of the neuron's receptive field. For the $\mathrm{H} 1$ neuron, the reference/test grating covered the entire horizontal width of the screen with a vertical extent of $26^{\circ}$, whereas for the V1 neuron the reference/test grating covered the entire vertical dimension of the screen with a horizontal extent of $30^{\circ}$. The adapting stimulus was either shown in the same part of the screen as the reference/test stimulus or it covered the remaining parts of the screen excluding the central reference/test area (Fig. 3: same area/separated area, respectively). When different parts of the receptive field were stimulated during testing and adapting periods, the adapting stimulus consisted of two areas flanking the region of the reference/test stimuli. In a series of pre-tests this stimulus was balanced in its size to elicit approximately the same initial response as the reference stimulus. This calibration ensures that similar levels of activity are elicited by the two types of adapting stimuli. Thus, the resulting activity-dependent adaptation, which is based on cell-intrinsic changes, is expected to be equally strong (Fig. 3A and B, top right).

All stimuli consisted of sinusoidal gratings with a wavelength of $8^{\circ}$ and a Michelson contrast of 0.95 , drifting at a temporal frequency of $4 \mathrm{~Hz}$ in the preferred direction of the neuron, i.e. back-to-front for $\mathrm{H} 1$ neurons and downward for V1 neurons. The reference stimulus was presented for $2 \mathrm{~s}$. Then, the screen switched to mean luminance for $0.2 \mathrm{~s}$ followed by the adapting stimulus presented for $10 \mathrm{~s}$. After the adapting stimulus, the screen was again blanked at mean luminance for $0.2 \mathrm{~s}$ followed by the test stimulus.

\section{Velocity discontinuities}

The stimulus used to characterize the impact of adaptation on neuronal responses to stimulus discontinuities has been described by Kurtz et al. (2009b). Briefly, a drifting sinusoidal grating was shown for $7 \mathrm{~s}$ at a constant baseline velocity as indicated in Fig. 4, moving in the neuron's preferred direction. This continuous motion was interrupted by eight brief periods during which the velocity was transiently
A

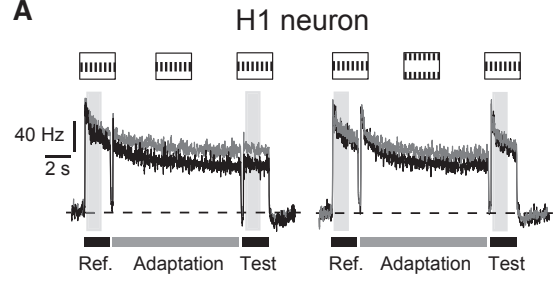

B
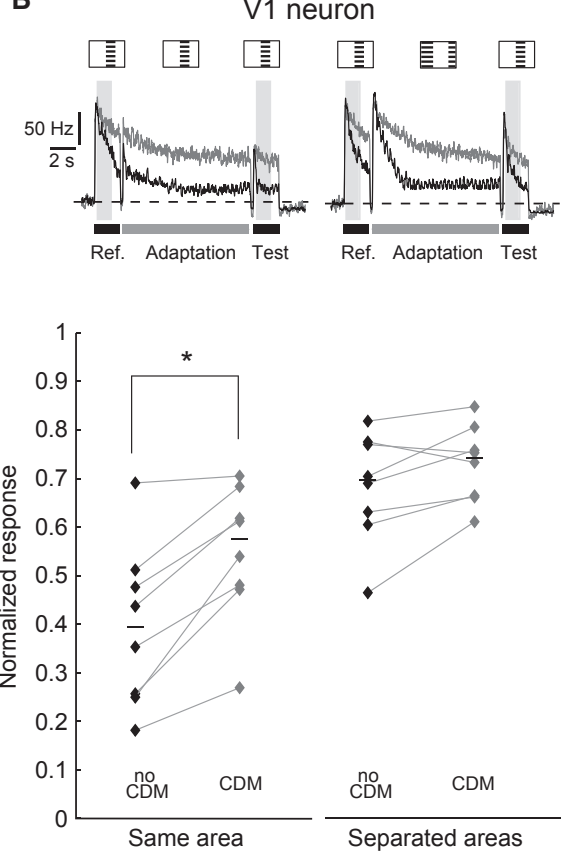

FIG. 3. Local vs. global effects of chlordimeform (CDM) on motion adaptation. (A) Top, representative responses of a H1 neuron to a sinusoidal grating drifting at constant velocity in the preferred direction (back-to-front) before (black) and after (grey) CDM application. Adapting and reference/test stimuli were either displayed in the same region of the receptive field (left) or in spatially separated regions (right), see Materials and methods for details. The resting activity was subtracted from the responses. Bottom, responses of the $\mathrm{H} 1$ neurons were averaged in a 1-s time window starting $100 \mathrm{~ms}$ after stimulus onset, as indicated by the shaded area in the top traces, and normalized to the reference stimulus. Each pair of symbols represents data of one fly obtained before (black) and after (grey) CDM application $(N=7)$. (B) Top, representative responses of a V1 neuron to the same stimulus paradigm (the grating was moving downward, the neuron's preferred direction). Bottom, responses of the V1 neurons quantified in the same way as for the H1 neuron $(N=8)$. The black horizontal bars depict the median across all flies. The asterisks represent statistical significance at the $5 \%$ significance level (paired $t$-test). 
A
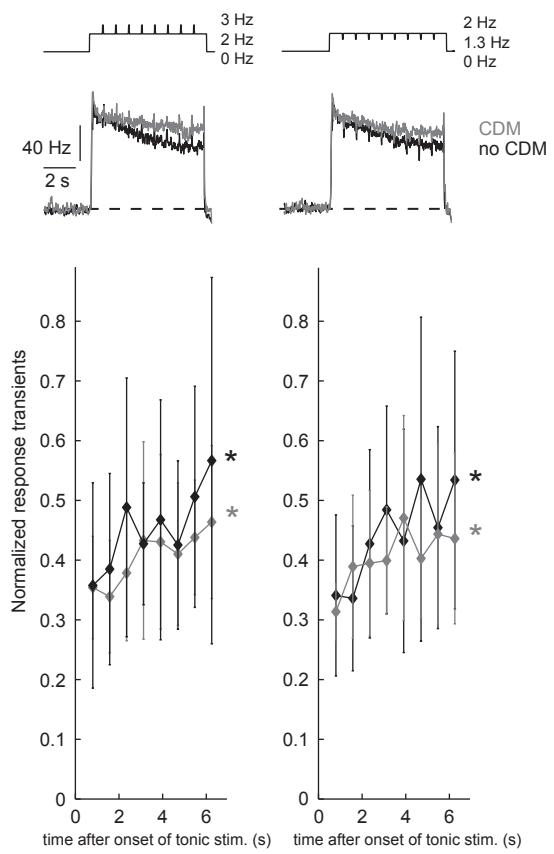

Velocity increments

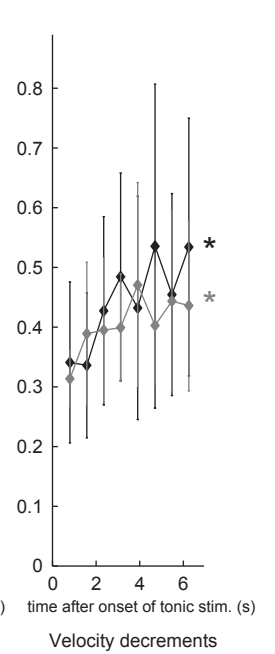

B
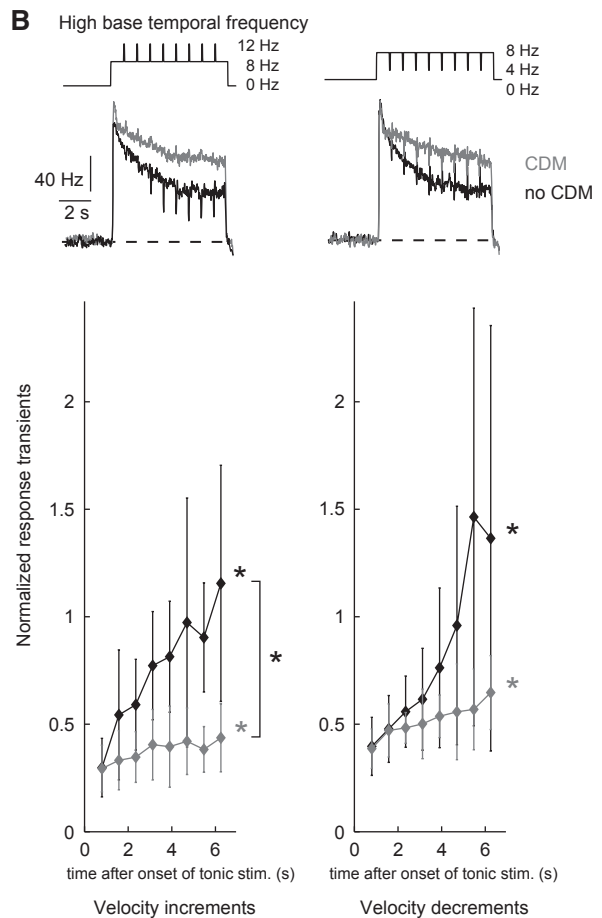

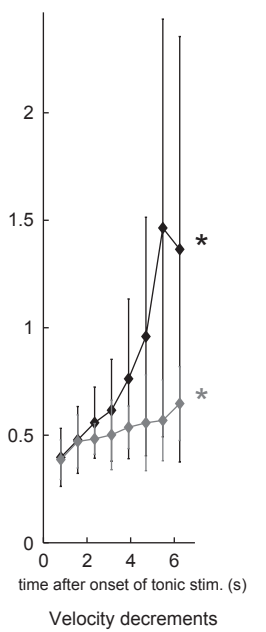

FIG. 4. Responses of $\mathrm{H} 1$ neurons to discontinuities in motion velocity. (A) Top, representative response of a H1 neuron to a grating moving at a temporal frequency (TF) of $2 \mathrm{~Hz}$ interspersed with eight 50-ms periods of an increment (TF $3 \mathrm{~Hz}$; left) or decrement (TF $1.3 \mathrm{~Hz}$; right) in temporal frequency. Bottom, normalized responses to the transient changes in the temporal frequency of the stimulus. (B) Top, representative responses of the same $\mathrm{H} 1 \mathrm{neuron}$ to increments (12 $\mathrm{Hz}$; left) or decrements $(4 \mathrm{~Hz}$; right) in the temporal frequency based on $8 \mathrm{~Hz}$ background temporal frequency. The grey asterisks indicate a significant steady increase in the response to the discontinuity with continuous stimulation (Mann-Kendall non-parametric trend test, $P<5 \%, N=6$ ). The black asterisk represents a significant difference in the response to the last discontinuity before and after CDM application (paired $t$-test, $P<5 \%$ ).

switched to a value above or below baseline velocity. The stimulus discontinuities were presented every $780 \mathrm{~ms}$ for $50 \mathrm{~ms}$; further details are shown in Fig. 4.

\section{Data analysis}

Data were evaluated offline using custom analysis routines written in MATLAB 2010b (The MathWorks, Natick, MA, USA). The number of neurons is denoted by ' $N$ '. We applied the Student's $t$-test for paired samples to test for statistically significant differences between the untreated and the CDM-treated condition, considering a significance level of $P<5 \%$. The non-parametric rank-based Mann-Kendall test was used to determine the statistically significant development of data trends over time. If not stated otherwise, spike rates will be given as mean \pm standard deviation.

\section{Results}

The octopamine agonist CDM has been shown to increase spontaneous as well as stimulus-induced activity in a similar way as walking or flight activity (Longden \& Krapp, 2009, 2010; Jung et al., 2011). CDM was also shown to modify the time course of the motion responses, in particular during stimulation with fast motion velocities, which elicited highly transient responses in the absence of CDM and more sustained responses after CDM application (Longden \& Krapp, 2010; Jung et al., 2011). The overall enhancement of neural responses by $\mathrm{CDM}$ as well as the change in velocity tuning might therefore, at least partially, result from a reduction of motion adaptation. In the present study, we used specific stimulation paradigms to investigate: (i) which of the different adaptation components are affected by CDM; (ii) at which stage of the visual motion pathway CDM is effective; (iii) and how the modulation of adaptive properties affects neuronal sensitivity for changes in stimulus velocity.

\section{Modulation of contrast gain adaptation by CDM}

In hoverfly HS neurons, a class of graded-potential LPTCs, a major reason for the decline of neuronal activity during motion adaptation has been attributed to a strong reduction in contrast sensitivity (Harris et al., 2000). To analyse the cellular mechanisms underlying octopaminergic regulation of LPTC activity we analysed whether the alteration of contrast sensitivity due to adaptation is affected by CDM. We used sinusoidal gratings drifting in the preferred direction of the V1 or H1 neurons in a sequence of reference, adaptation and test stimuli, all of which covered the same area of the receptive field (Fig. 1A and E). The contrast of the adapting stimulus was held fixed at the maximum contrast that could be delivered by the CRT display. For the reference and test stimuli a contrast value was chosen pseudorandomly out of a range of 12 contrast levels in each trial. Thus, contrast sensitivity can be determined (see also Harris et al., 2000) for the unadapted state, based on the responses to the reference stimuli, and for the adapted state, based on the responses to the test stimuli.

In accordance with Longden \& Krapp $(2009,2010)$ and Jung et al. (2011), we observed an increase in spontaneous spike rate in H1 and V1 neurons after CDM application (H1 before CDM: $23 \pm 8.9 \mathrm{~Hz}$; after CDM: $29 \pm 8.6 \mathrm{~Hz}, P=0.011, N=24$; V1 before CDM: $20 \pm 5.7 \mathrm{~Hz}$; after CDM: $42 \pm 13.3 \mathrm{~Hz}, P=0.00000001, N=24)$. 
To facilitate the comparison of the responses before and after CDM application, we subtracted the mean spontaneous spike rate from the response traces. Adaptation was generally stronger in the V1 neurons than in the H1 neuron (cf. Fig. 1A and E). Consequently, the effect of $\mathrm{CDM}$ appears to be more pronounced in V1 neurons. One possible explanation for the difference in adaptation strength between the two types of LPTCs is the difference in their input structure. Unlike the H1 neuron, the V1 neuron receives its input not directly from local motion-detecting neurons but from other types of LPTCs. Thus, an additional presynaptic layer can contribute to overall adaptation of the V1 neuron (see also Beckers et al., 2007; Kalb et al., 2008). Figure $1 \mathrm{~B}$ and $\mathrm{F}$ displays the contrast-response functions of $\mathrm{H} 1$ and V1 neurons, respectively, before (solid symbols) and after (open symbols) adaptation. In the unadapted state, the contrast-response functions had a similar sigmoid shape before and after CDM application. In general, slightly larger response amplitudes were present after CDM application, which in the H1 neuron was not significantly different from those obtained under control conditions (Fig. 1B, solid symbols). In V1 neurons, CDM application significantly increased the responses to the reference stimuli at contrasts exceeding 0.25 (Fig. 1F, solid symbols). Following adaptation, motion-induced responses were attenuated in the presence and absence of CDM, in particular at low contrasts (Fig. 1B and F; cf. solid and open symbols), corroborating that similar to hoverfly HS neurons (Harris et al., 2000) contrast sensitivity in blowfly H1 and V1 neurons is also reduced by motion adaptation. However, after administration of CDM, the impact of adaptation on the contrastresponse functions was less pronounced. The effect of CDM is particularly evident when comparing the data points and the corresponding sigmoid fits for the conditions with and without CDM (Fig. 1B and F; cf. black and grey data points). In the unadapted state the curves obtained under these two conditions are close to each other, and the error bars of the corresponding data points overlap at all (Fig. 1B, solid data points for $\mathrm{H} 1$ neurons) or some contrast values (Fig. 1F, solid data points for V1 neurons). In the adapted state, the contrast sensitivity curves for the data obtained before and after application of CDM differ much more (Fig. 1B and F, open data points), resulting in an overlap of the error bars of corresponding data points only in the low-contrast range. To further quantify the effect of $\mathrm{CDM}$ on the adaptation-induced reduction of contrast sensitivity, we first normalized the data obtained from individual neurons to the unadapted responses measured at the highest contrast. To illustrate the difference in adaptation properties induced by CDM, we plotted the differences in the spike rates between the unadapted and adapted responses for data obtained before and after CDM application (see Fig. $1 \mathrm{C}$ for $\mathrm{H} 1$ neurons and Fig. $1 \mathrm{G}$ for $\mathrm{V} 1$ neurons). In the lowcontrast range $\mathrm{CDM}$ did not change the effect of adaptation on the relative strength of the neuronal response. However, at contrasts exceeding 0.5 (H1) or 0.3 (V1), the effect of adaptation is significantly attenuated after CDM application. This finding indicates that modulation of neuronal sensitivity by CDM is at least partially mediated by a reduction of contrast gain adaptation.

To quantify the shift of the contrast-response function along the $x$-axis (the 'contrast gain reduction' according to Harris et al., 2000), we determined the contrast that elicited $25 \%\left(C_{25}\right)$ of the maximum response, as obtained from the fit to the unadapted responses before CDM application (see Fig. 1D for H1 neurons and Fig. 1H for V1 neurons). Before and after CDM application we observed for all neurons a significant shift of the $C_{25}$ towards higher contrasts (Fig. 1Di, $P=0.001$ before, $P=0.003$ after CDM, $N=6$ for $\mathrm{H} 1$ neurons; Fig. $1 \mathrm{Hi}, P=0.012$ before, $P=0.021$ after CDM, $N=5$ for V1 neurons). CDM application resulted in a diminished rightward shift of the $C_{25}$ in five out of six H1 neurons (Fig. 1Dii). The same effect was observed in V1 neurons (Fig. 1Hii), where in all investigated neurons CDM application led to a significantly smaller contrast gain reduction $(P=0.045)$.

The adaptation protocols used so far did not allow us to assess whether the effect of adaptation on contrast sensitivity results from changes of physiological properties intrinsic to the $\mathrm{H} 1$ neuron (or V1 neuron) or from changes localized at more peripheral processing stages. In a previous study on motion adaptation in hoverfly HS neurons (Harris et al., 2000), the changes in contrast sensitivity could be broken down into several components. One of these components was independent of LPTC activity and was therefore concluded to result from changes in the periphery. This type of adaptation, which was not only elicited by motion in the LPTC's preferred direction, but also by motion in the null direction and by motion orthogonal to the preferred/null axis, is expected to remain locally restricted within the LPTC's receptive field (Harris et al., 2000; Nordström \& O'Carroll, 2009). This adaptation component is reflected by a rightward shift in the contrast-response function, similar to what we found in $\mathrm{H} 1$ and V1 neurons (Fig. 1). To analyse whether the modulation of contrast gain reduction by $\mathrm{CDM}$ is independent from the activity of $\mathrm{V} 1$ or $\mathrm{H} 1$ neurons, we adapted with motion in a direction orthogonal to their respective preferred direction (Fig. 2A and E). Similar to the protocol applied to obtain the data shown in Fig. 1, contrast sensitivity was assessed by using reference and test stimuli with various contrast values moving in the preferred direction.

In general, the adaptation affected the contrast-response functions less when induced by orthogonal motion than by preferred-direction motion (Figs 1A and E, and 2A and E). However, shifts of the adapted curves towards higher contrasts relative to the unadapted curves as well as slight reductions in output range were still present after orthogonal adaptation (Fig. 2B and F). Moreover, these effects of adaptation are weaker following application of CDM (Fig. 2B and F; cf. black and grey symbols). Following CDM application, the normalized adaptation-induced differences in the response amplitudes were significantly smaller than before CDM application at contrasts exceeding $30 \%$ for the $\mathrm{H} 1$ and for the V1 neuron (Fig. 2C and G). The effect of CDM on contrast adaptation after orthogonal adaptation appears not to differ in a qualitative manner from that induced by preferred-direction adaptation (Figs $1 \mathrm{C}$ and G, and 2C and G). Similar to the observed contrast gain reduction after preferred direction adaptation, we observed in the $\mathrm{H} 1$ neuron as well as in the V1 neuron a significant shift of the contrast-response function toward higher contrasts after orthogonal adaptation (Fig. 2Di, $P=0.0001, N=9$ for H1; Fig. 2Hi, $P=0.0002, N=9$ for V1). This shift, as quantified by the $C_{25}$ value, was slightly less pronounced after orthogonal adaptation than after preferred-direction adaptation (Figs 1D and H, and 2D and $\mathrm{H}$ ). After CDM application, orthogonal motion adaptation significantly reduced $C_{25}$ values (Fig. 2Di, $P=0.0003, N=9$ for H1; Fig. $2 \mathrm{Hi}, P=0.0002, N=9$ for V1). However, similar to what has been found after preferred-direction adaptation, the magnitude of this contrast gain reduction was weaker after CDM application than before (Fig. 2Dii, $P=0.004, N=9$ for H1; Fig. 2Hii, $P=0.04$, $N=9$ for V1). These results demonstrate that CDM affects adaptation of LPTCs by modifying their contrast sensitivity even if the adapting stimulus elicits no or at most very weak activity of the LPTCs. Thus, CDM most likely affects adaptation of contrast sensitivity in neurons presynaptic to LPTCs. Due to the structure of the presynaptic neuropils, consisting of numerous retinotopically arranged local motion-processing neurons, the modulation of contrast sensitivity by $\mathrm{CDM}$ is expected to affect the response properties of individual LPTCs only locally. 


\section{Impact of CDM on local and global adaptation}

To examine directly whether the modulation of the response properties of LPTCs by CDM results from an impact on local or global adaptation, we used two adaptation protocols that differed regarding stimulus location (analogous to Maddess \& Laughlin, 1985; Nordström \& O'Carroll, 2009). In one protocol the motion stimuli for adapting the neuron were presented in the same area as the stimuli used to test the strength of motion adaptation. In this protocol all components of adaptation are effective, regardless of their localization. In the second protocol, adapting stimuli were presented in a different part of the receptive field of the LPTC under study than the reference and adapting stimuli. In this protocol the responses to the test stimuli are affected by adaptation components intrinsic to LPTCs only, because they integrate inputs from different parts of the visual field. Thus, this type of adaptation acts in a global manner, because it is transferred from the adapted parts of the visual field to those parts of the visual field that belong to the receptive field of the LPTC, but were not stimulated during adaptation. In contrast, adaptation of presynaptic neurons will remain local, because each of the presynaptic neurons receives input only from a confined part of the visual field. Therefore, local adaptation will remain ineffective when adapting and testing is performed in different parts of the receptive field of an LPTC.

When testing and adapting in the same part of the receptive field, the application of CDM attenuated motion adaptation in $\mathrm{H} 1$ and V1 neurons, as is visible in a higher tonic response level at the end of the adapting period and in the larger response to the test stimulus (Fig. 3A and B, top left). When testing and adapting in different parts of the receptive field, a difference between the CDM and the control results is, as with the previous stimulus protocol, visible in the time course of the response during the adapting stimulus (Fig. 3A and B, top right). However, either no prominent difference between control and CDM is immediately visible (in $\mathrm{H} 1$ neurons, see Fig. 3A, top right), or the difference between control and CDM is about equally pronounced during the test period as during the reference period (in V1 neurons, see Fig. 3B, top right). The difference in the effects of $\mathrm{CDM}$ between the two stimulus protocols demonstrates that the impact of CDM on adaptation critically depends on whether adapting and testing is performed in the same part or in different parts of the receptive field.

We quantified the mean responses of individual neurons to the reference and test stimuli in the 'same area' and 'separated area' stimulus paradigms (Fig. 3A and B, bottom). For normalization the mean responses of each neuron were divided by the mean response to the reference stimulus. Consistent with previous results (Nordström \& O'Carroll, 2009), significant adaptation was present in either case when adapting and testing in the same part of the receptive field as well as when adapting and testing in separate parts. This corroborates previous conclusions that adaptation of LPTCs consists of presynaptic as well as intrinsic components (Harris et al., 2000; Kurtz et al., 2000, 2009a; Kurtz, 2007; Nordström \& O'Carroll, 2009). However, in H1 and V1 neurons adapting and testing in the same part of the receptive field induced significantly stronger adaptation compared with the condition when spatially separated parts of the receptive field were stimulated during adapting and testing (Fig. 3A, bottom, $P=0.000002, N=7$ for $\mathrm{H} 1$ neurons; Fig. $3 \mathrm{~B}$, bottom, $P=0.0007$, $N=8$ for $\mathrm{V} 1$ neurons). Both in $\mathrm{H} 1$ and $\mathrm{V} 1$ neurons a significant CDM-mediated attenuation of motion adaptation was present when adapting and test stimuli were displayed in the same part of the receptive field (Fig. 3A, bottom left, $P=0.003, N=7$ for $\mathrm{H} 1$ neurons; Fig. 3B, bottom left, $P=0.03, N=8$ for V1 neurons). In contrast, when showing adapting and test stimuli in spatially separated parts of the receptive field, CDM induced only moderate, statistically non-significant differences in adaptation (Fig. 3A, bottom right, $P=0.1, N=7$ for $\mathrm{H} 1$ neurons; Fig. $3 \mathrm{~B}$, bottom right, $P=0.2$, $N=8$ for $\mathrm{V} 1$ neurons). This difference between our two stimulus protocols suggests that CDM predominantly or exclusively affects local adaptation, which is most likely generated by changes in the sensitivity of neurons with small receptive fields presynaptic to LPTCs. In contrast, it is unlikely that CDM has a strong effect on intrinsic, activity-dependent adaptation of LPTCs, which would act in a global manner. A strong direct effect of CDM on LPTCs would only be compatible with our results if CDM modulated adaptation by changing the sensitivity of local postsynaptic sites on the retinotopically organized dendrites of LPTCs. Such a modulation of synaptic input sites would, similar to changes in presynaptic neurons, produce effects that remain locally confined.

\section{Impact of CDM on the neuronal responsiveness to discontinuities in stimulus velocity}

Recently, motion adaptation was shown to enhance the responses of the $\mathrm{H} 1$ neuron to sudden changes of various stimulus parameters during continuous stimulation (Kurtz et al., 2009b). This property, which was suggested to be functionally relevant in the context of enhancing the detection of motion contrast (Maddess \& Laughlin, 1985), might be affected by CDM, because, as shown above, motion adaptation is attenuated after application of CDM.

We quantified the responses of $\mathrm{H} 1$ neurons to sudden changes in velocity during continuous stimulation before and after application of CDM by using a stimulus protocol similar to that applied in Kurtz et al. (2009b). Brief increments or decrements in velocity were added to a baseline velocity of a grating at various time points after motion onset (Fig. 4A, top). When stimulated with a low baseline velocity, the neurons responded to velocity increments and decrements with an increase and decrease in spike rate, respectively (Fig. 4A). The sign of the responses to the velocity changes suggests that the baseline velocity was below the maximum of the bell-shaped velocity tuning curve (Maddess \& Laughlin, 1985). The overall baseline spike rate decreased to an adapted level, which was about $2 / 3$ of the initial response. We quantified the responses to the sudden changes in stimulus velocity by calculating the difference between the maximum and minimum in a $150-\mathrm{ms}$ time window starting at stimulus onset. These values were divided by the average spike rate in a 50-ms time window immediately preceding the onset of the corresponding velocity discontinuity to obtain a measure for the response to the velocity discontinuity relative to the current activity level (for details, see Kurtz et al., 2009b).

Before CDM application, the relative responses to the velocity transients increased significantly during sustained motion stimulation (Fig. 4A, bottom, black lines; Mann-Kendall test; velocity increments $P=0.034$, velocity decrements $P=0.035, N=7$ ). After CDM application the baseline response decreased with continuous stimulation, as expected, less than without CDM. Although the responses to the velocity discontinuities were not as prominent as before CDM application, their amplitude still increased significantly relative to the current activity level (Fig. 4A, bottom, grey lines; Mann-Kendall test; velocity increments $P=0.019$, velocity decrements $P=0.019$, $N=7)$.

We next tested the response of $\mathrm{H} 1$ neurons to velocity discontinuities during baseline motion with a velocity higher than the neuron's steady-state velocity optimum. As expected, the neurons responded with a decrease in spike rate to a velocity increment and with an 
increase in spike rate to a velocity decrement (Fig. 4B). Without CDM the overall background spike rate decreased rapidly to approximately $1 / 2$ of the initial response. However, the relative amplitudes of the responses to the transient changes in stimulus velocity (quantified as described above) increased significantly with continuous motion adaptation (Fig. 4B, bottom; Mann-Kendall test; velocity increments $P=0.002$, velocity decrements $P=0.002, N=7$ ). The effect of $\mathrm{CDM}$ on the time course of the response was stronger compared with the lower baseline velocity shown in Fig. 4A. After CDM application the responses to the high background velocity stayed at a relatively high level of about $2 / 3$ of the initial response. In addition, the transients in response to the velocity discontinuities were less distinct, in particular for velocity increments. However, we still observed a significant increase of the relative response transients during motion adaptation (Mann-Kendall test; velocity increments $P=0.018$, velocity decrements $P=0.001, N=7$ ). In some of the neurons, the sign of the response transients was reversed after CDM application. For example, the response of the neuron shown in Fig. $4 \mathrm{~B}$ to a velocity increment was a decrease in spike rate before CDM application but an increase in spike rate after CDM application. However, as these effects were not consistent across our sample of neurons, further tests would be necessary to assess how CDM affects the response properties of LPTCs under dynamic stimulus conditions.

In summary, as shown before (Kurtz et al., 2009b), relative responses of $\mathrm{H} 1$ neurons to sudden changes in the stimulus velocity increased during motion adaptation regardless of whether the baseline velocity was below or above the neuron's velocity optimum. Although CDM application resulted in an attenuation of motion adaptation and thus in less pronounced transient responses to sudden velocity changes, a significant enhancement of the sensitivity to velocity discontinuities during motion adaptation appears to be preserved.

\section{Discussion}

Changes of the behavioural state of an animal have been shown to alter visual processing in vertebrate as well as invertebrate species (mice: Niell \& Stryker, 2010; Andermann et al., 2011; birds: McArthur \& Dickman, 2011; flies: Rosner et al., 2009, 2010; Chiappe et al., 2010; Maimon et al., 2010; Jung et al., 2011). In locusts, the biogenic amine octopamine is released in high quantities during flight (Goosey \& Candy, 1980) and, in locusts as well as in other invertebrates, has been shown to affect metabolism (Wegener, 1996) and the processing of visual information (Bacon et al., 1995; Erber \& Kloppenburg, 1995; Chyb et al., 1999; Battelle et al., 1999; Longden \& Krapp, 2009, 2010). In this study, we used the octopamine agonist CDM to mimic a behavioural high-activity state similar to what the fly experiences during locomotion (Jung et al., 2011). We focussed on the effects of CDM on various aspects of motion adaptation to explain how stimulus-response functions of fly LPTCs are modulated by the behavioural state of the animal.

\section{Impact of CDM on contrast gain adaptation and its implications for velocity tuning}

In the present paper we demonstrate that the reduction of contrast sensitivity of fly LPTCs, one of the hallmarks of motion adaptation (Harris et al., 2000), is attenuated by CDM. This result can explain why responses to motion are enhanced when the fly is flying or walking compared with periods of resting (Longden \& Krapp, 2011). The state-dependent difference in the strength of contrast gain reduction would also manifest itself as a difference in the time course of the motion response. Consistent with this prediction, typical differences in the time course of motion responses were observed after CDM application and after a change of the activity state (Longden \& Krapp, 2010; Jung et al., 2011). In particular, the response to fast pattern motion, which consists of a sharp transient in spike rate upon motion onset followed by a gradual decline to a much lower steadystate level, was found to settle on a markedly higher steady-state after application of CDM as well as after transition from rest to flight (Longden \& Krapp, 2010; Jung et al., 2011; Figs 1, 3 and 4). In a computational model employing the well-established HassensteinReichardt-type motion detectors, these state-dependent alterations in response properties were reproduced by corresponding adjustments of temporal filters of the motion detector (Jung et al., 2011). Statedependent temporal filtering also resulted in a shift of the steady-state temporal frequency tuning towards higher values, similar to what was experimentally observed in active compared with resting flies as well as after application of CDM (Chiappe et al., 2010; Jung et al., 2011; Fig. 4). Thus, the modelling approach provides a good phenomenological description for state-dependent alterations of visual motion processing, but it does, of course, not identify potential neuronal mechanisms.

Several arguments support the notion that the modulation of contrast gain adaptation, as induced in the present study by CDM application, forms a neuronal mechanism underlying state-dependent changes in temporal frequency tuning of LPTCs. Contrast gain adaptation and, correspondingly, the effect of CDM on adaptation stays locally confined to the adapted region in the receptive field of the H1 neuron and V1 neuron (Fig. 3). Thus, contrast gain adaptation most likely results from changes in the local input elements of the $\mathrm{H} 1$ neuron and the V1 neuron, and is therefore controlled by the temporal frequency of local brightness modulations rather than by the overall activity of these neurons. Contrast gain adaptation would therefore increase in strength with increasing temporal frequency of a drifting grating. This is consistent with a larger effect of CDM at higher temporal frequencies, as observed by Longden \& Krapp (2010). This difference would manifest itself as a shift in the steady-state temporal frequency tuning towards higher values, as was in fact observed after CDM application and after transition from rest to flight (Jung et al., 2011).

Moreover, a state-dependent difference in the time course of the response of LPTCs is present soon after motion onset. Contrast gain adaptation is fast enough to account for this difference in time courses, because it was recently shown to evolve after as few as several tens of milliseconds of stimulation (Nordström et al., 2011). Still, it is important to note that a significant state-dependent difference in the temporal frequency tuning was not detectable in a time window of $0.5 \mathrm{~s}$ beginning immediately with motion onset (Longden \& Krapp, 2010). The apparent contradiction between this result and those of other studies, in which later time windows were evaluated (Chiappe et al., 2010; Jung et al., 2011), is easily explained if it is considered that differences due to modulation of contrast gain adaptation, though fast, need some time of sustained motion stimulation to become observable.

\section{Cellular mechanisms underlying modulation of LPTC activity by $C D M$}

The cellular mechanisms by which octopamine exerts its effects are largely unknown, although there is some recent evidence that octopamine acts via G-protein-coupled receptors activating the $\mathrm{Ca}^{2+} /$ calmodulin and cAMP pathways (Roeder, 2005). In spider 
mechanosensory neurons, octopamine caused a persistent increase in excitability by activating calcium release from internal stores, thus modulating voltage-activated potassium channels via $\mathrm{Ca}^{2+} /$ calmodulin-dependent protein kinase II (Torkkeli et al., 2011).

Although effects of CDM on visual processing in flies were in all previous studies assessed on the basis of the LPTC activity, our results indicate that a major site of action of CDM lies presynaptic to LPTCs. In particular, adaptation by orthogonal motion, which does not elicit activity in the recorded LPTC, was affected by CDM (Fig. 2). Consistently, global adaptation, which spreads from one part of the receptive field of the LPTC to another, was modulated by CDM much less than adaptation that stays locally confined within the receptive field (Fig. 3). The involvement of neurons presynaptic to LPTCs in state-dependent regulation is also supported by the finding that the input resistance of LPTCs in Drosophila decreased during flight compared with rest (Maimon et al., 2010). Using genetic markers, several large octopaminergic neurons with extended arborizations in nearly all major regions of the optic lobe were recently identified in Drosophila, reaching from central brain areas to the lobula complex as well as the medulla, the neuropil regarded to be the major site of local motion processing presynaptic to LPTCs (Busch et al., 2009). Based on octopamine immunoreactivity, processes of octopaminergic neurons were also identified in the lobula complex of a blowfly, Phaenicia sericata (Sinakevitch \& Strausfeld, 2006).

The adaptation of contrast sensitivity of visual motion processing may not be the only process that undergoes octopaminergic modulation, because such changes in adaptation cannot easily explain the strong increase in spontaneous activity of LPTCs following the start of locomotor activity or CDM application (Longden \& Krapp, 2009; Jung et al., 2011). The notion that state-dependent changes of spontaneous and visually evoked activity are caused by separate cellular mechanisms is corroborated by the finding that these changes differ in their temporal profiles. Following the cessation of flight, the change in spontaneous activity recovered almost immediately whereas the change in visual sensitivity recovered exponentially with a time constant of 5-10 s (Maimon et al., 2010). The state-dependent changes in spontaneous activity are likely caused by synaptic input from neurons that change their activity immediately after (or even slightly before) transitions in locomotor state. The presence of such inputs to LPTCs was proposed in a study in which changes of locomotor state were assessed in tethered flies by monitoring the movements of halteres, the fly's gyroscopic sensors derived from its hind wings, which oscillate during flight and walking (Rosner et al., 2010).

\section{Functional significance of neuromodulatory control of contrast gain adaptation}

An octopamine-induced reduction of contrast gain adaptation during flight and the resulting maintenance of a fairly large response of LPTCs even in the presence of fast image velocities might be functionally beneficial, because image velocities are in general higher during locomotion than during rest. Too strong adaptation would then limit the neuronal capacity to encode modulations in stimulus intensity, in particular those leading to further reductions in spike rate. In contrast, when the fly is inactive, strong adaptation and the reduction in spontaneous neuronal activity might help reduce metabolic costs for neuronal signalling (Niven \& Laughlin, 2008). Similar state-dependent regulation of visually evoked activity, but no change in spontaneous activity or stimulus selectivity, has recently been demonstrated in mouse primary visual cortex (Niell \& Stryker, 2010).
In contrast, in a later study a slight shift of the velocity tuning towards higher values was found in addition to a general enhancement of somatic calcium signals in the primary visual cortex as well as in two downstream target areas (Andermann et al., 2011). In the vestibular system of pigeons, motion-sensitive neurons showed variable state dependence, with some neurons responding to simulated flight with increased spontaneous activity, and others with a response gain enhancement for rotational stimuli (McArthur \& Dickman, 2011). These comparisons indicate that the effect of state-dependent regulation might depend on the functional task of a brain region or of particular types of neurons. Although state-dependent regulation appears to be qualitatively similar across different LPTCs, future studies might show whether task-related differences between individual LPTCs exist, and how behavioural states affect other stages of the fly's visuomotor system.

\section{Acknowledgements}

The authors thank M. Egelhaaf for numerous insightful discussions and comments on the manuscript. The helpful comments of two anonymous reviewers on an earlier version of the manuscript are gratefully acknowledged.

\section{Abbreviations}

CDM, chlordimeform; LPTC, lobula plate tangential cell.

\section{References}

Andermann, M.L., Kerlin, A.M., Roumis, D.K., Glickfeld, L.L. \& Reid, R.C. (2011) Functional specialization of mouse higher visual cortical areas. Neuron, 72, 1025-1039.

Bacon, J.P., Thompson, K.S. \& Stern, M. (1995) Identified octopaminergic neurons provide an arousal mechanism in the locust brain. J. Neurophysiol., 74, 2739-2743.

Battelle, B.A., Calman, B.G. \& Hart, M.K. (1999) Cellular distributions and functions of histamine, octopamine, and serotonin in the peripheral visual system, brain, and circumesophageal ring of the horseshoe crab Limulus polyphemus. Microsc. Res. Tech., 44, 70-80.

Beckers, U., Egelhaaf, M. \& Kurtz, R. (2007) Synapses in the fly motion-vision pathway: evidence for a broad range of signal amplitudes and dynamics. $J$. Neurophysiol., 97, 2032-2041.

Borst, A., Haag, J. \& Reiff, D.F. (2010) Fly Motion Vision. Annu. Rev. Neurosci., 33, 49-70.

Busch, S., Selcho, M., Ito, K. \& Tanimoto, H. (2009) A map of octopaminergic neurons in the Drosophila brain. J. Comp. Neurol., 513, 643-667.

Chiappe, M.E., Seelig, J.D., Reiser, M.B. \& Jayaraman, V. (2010) Walking modulates speed sensitivity in Drosophila motion vision. Curr. Biol., 20, $1470-1475$.

Chyb, S., Hevers, W., Forte, M., Wolfgang, W.J., Selinger, Z. \& Hardie, R.C. (1999) Modulation of the light response by cAMP in Drosophila photoreceptors. J. Neurosci., 19, 8799-8807.

Costa, L.G., Olibet, G. \& Murphy, S.D. (1988) Alpha 2-adrenoceptors as a target for formamidine pesticides: in vitro and in vivo studies in mice. Toxicol. Appl. Pharmacol., 93, 319-328.

Eckert, H. (1980) Functional properties of the H1-neurone in the third optic ganglion of the blowfly, Phaenicia. J. Comp. Physiol. A., 135, 29-39.

Egelhaaf, M. \& Borst, A. (1989) Transient and steady-state response properties of movement detectors. J. Opt. Soc. Am. A, 6, 116-127.

Egelhaaf, M., Kern, R., Krapp, H.G., Kretzberg, J., Kurtz, R. \& Warzecha, A.K. (2002) Neural encoding of behaviourally relevant visual-motion information in the fly. Trends Neurosci., 25, 96-102.

Erber, J. \& Kloppenburg, P. (1995) The modulatory effects of serotonin and octopamine in the visual system of the honey bee (Apis mellifera L.). $J$. Comp. Physiol. A., 176, 111-118.

Evans, P.D. \& Gee, J.D. (1980) Action of formamidine pesticides on octopamine receptors. Nature, 287, 60-62.

Franceschini, N. (1975) Sampling of visual environment by the compound eye of the fly: fundamentals and applications. In Snyder, A.W. \& Menzel, R. (Eds), Photoreceptor Optics. Springer, New York, pp. 98-125. 
Goosey, M.W. \& Candy, D.J. (1980) Effects of D- and L-octopamine and of pharmacological agents on the metabolism of locust flight muscle. Biochem. Soc. Trans., 8, 532-533.

Harris, R.A., O'Carroll, D.C. \& Laughlin, S.B. (2000) Contrast gain reduction in fly motion adaptation. Neuron, 28, 595-606.

Hausen, K. (1982) Motion sensitive interneurons in the optomotor system of the fly: II. The horizontal cells: receptive field organization and response characteristics. Biol. Cybern., 46, 67-79.

Hausen, K. (1984). The lobula-complex of the fly: structure, function and significance in visual behaviour. In Ali, M.A. (Ed.), Photoreception and Vision in Invertebrates. Plenum Press, New York, London, pp. 523-559.

Hennig, P., Kern, R. \& Egelhaaf, M. (2011) Binocular integration of visual information: a model study on naturalistic optic flow processing. Front. Neural Circuits, 5, 4

Hiripi, L., Nagy, L. \& Hollingworth, R.M. (1999) In vitro and in vivo effects of formamidines in locust (Locusta migratoria migratorioides). Acta Biol. Hung., 50, 81-87.

Hollingworth, R.M. \& Murdock, L.L. (1980) Formamidine pesticides: octopamine-like actions in a firefly. Science, 208, 74-76.

Horstmann, W., Egelhaaf, M. \& Warzecha, A.K. (2000) Synaptic interactions increase optic flow specificity. Eur. J. Neurosci., 12, 2157-2165.

Jung, S.N., Borst, A. \& Haag, J. (2011) Flight activity alters velocity tuning of fly motion-sensitive neurons. J. Neurosci., 31, 9231-9237.

Kalb, J., Egelhaaf, M. \& Kurtz, R. (2008) Adaptation of velocity encoding in synaptically coupled neurons in the fly visual system. J. Neurosci., 28, $9183-$ 9193.

Karmeier, K., Krapp, H.G. \& Egelhaaf, M. (2003) Robustness of the tuning of fly visual interneurons to rotatory optic flow. J. Neurophysiol., 90, 16261634

Krapp, H.G., Hengstenberg, R. \& Egelhaaf, M. (2001) Binocular contributions to optic flow processing in the fly visual system. J. Neurophysiol., 85, 724734.

Kurtz, R. (2007) Direction-selective adaptation in fly visual motion-sensitive neurons is generated by an intrinsic conductance-based mechanism. Neuroscience, 146, 573-583.

Kurtz, R. (2011) Adaptive encoding of motion information in the fly visual system. In Barth, F.G., Humphrey, J.A.C. \& Srinivasan, M.V. (Eds), Frontiers in Sensing: From Biology to Engineering. Springer, Wien, New York, pp. 115-128.

Kurtz, R., Dürr, V. \& Egelhaaf, M. (2000) Dendritic calcium accumulation associated with direction-selective adaptation in visual motion-sensitive neurons in vivo. J. Neurophysiol., 84, 1914-1923.

Kurtz, R., Warzecha, A.K. \& Egelhaaf, M. (2001) Transfer of visual motion information via graded synapses operates linearly in the natural activity range. J. Neurosci., 21, 6957-6966.

Kurtz, R., Beckers, U., Hundsdörfer, B. \& Egelhaaf, M. (2009a) Mechanisms of after-hyperpolarization following activation of fly visual motion-sensitive neurons. Eur. J. Neurosci., 30, 567-577.
Kurtz, R., Egelhaaf, M., Meyer, H.G. \& Kern, R. (2009b) Adaptation accentuates responses of fly motion-sensitive visual neurons to sudden stimulus changes. Proc. Biol. Sci., 276, 3711-3719.

Longden, K.D. \& Krapp, H.G. (2009) State-dependent performance of opticflow processing interneurons. J. Neurophysiol., 102, 3606-3618.

Longden, K.D. \& Krapp, H.G. (2010) Octopaminergic modulation of temporal frequency coding in an identified optic flow-processing interneuron. Front. Syst. Neurosci., 4, 153.

Longden, K.D. \& Krapp, H.G. (2011) Sensory neurophysiology: motion vision during motor action. Curr. Biol., 21, R650-R652.

Maddess, T. \& Laughlin, S.B. (1985) Adaptation of the motion-sensitive neuron $\mathrm{H} 1$ is generated locally and governed by contrast frequency. Proc. Biol. Sci., 225, 251-275.

Maimon, G. (2011) Modulation of visual physiology by behavioral state in monkeys, mice, and flies. Curr. Opin. Neurobiol., 21, 559-564.

Maimon, G., Straw, A.D. \& Dickinson, M.H. (2010) Active flight increases the gain of visual motion processing in Drosophila. Nat. Neurosci., 13, 393-399.

McArthur, K.L. \& Dickman, J.D. (2011) Behavioral state modulates the activity of brainstem sensorimotor neurons. J. Neurosci., 31, 16700-16708.

Niell, C.M. \& Stryker, M.P. (2010) Modulation of Visual Responses by Behavioral State in Mouse Visual Cortex. Neuron, 65, 472-479.

Niven, J.E. \& Laughlin, S.B. (2008) Energy limitation as a selective pressure on the evolution of sensory systems. J. Exp. Biol., 211, 1792-1804.

Nordström, K. \& O'Carroll, D.C. (2009) The motion after-effect: local and global contributions to contrast sensitivity. Proc. Biol. Sci. 276, 1545-1554.

Nordström, K., de Miguel, I.M. \& O'Carroll, D.C. (2011) Rapid contrast gain reduction following motion adaptation. J. Exp. Biol., 214, 4000-4009.

Rind, F.C., Santer, R.D. \& Wright, G.A. (2008) Arousal facilitates collision avoidance mediated by a looming sensitive visual neuron in a flying locust. J. Neurophysiol., 100, 670-680.

Roeder, T. (2005) Tyramine and octopamine: ruling behavior and metabolism. Annu. Rev. Entomol., 50, 447-477.

Rosner, R., Egelhaaf, M., Grewe, J. \& Warzecha, A.K. (2009) Variability of blowfly head optomotor responses. J. Exp. Biol., 212, 1170-1184.

Rosner, R., Egelhaaf, M. \& Warzecha, A.K. (2010) Behavioural state affects motion-sensitive neurones in the fly visual system. J. Exp. Biol., 213, 331338.

Sinakevitch, I. \& Strausfeld, N.J. (2006) Comparison of octopamine-like immunoreactivity in the brains of the fruit fly and blow fly. J. Comp. Neurol. 494, 460-475.

Stern, M. (2009) The PM1 neurons, movement sensitive centrifugal visual brain neurons in the locust: anatomy, physiology, and modulation by identified octopaminergic neurons. J. Comp. Physiol. A., 195, 123-137.

Torkkeli, P.H., Panek, I. \& Meisner, S. (2011) $\mathrm{Ca}^{2+} /$ calmodulin-dependent protein kinase II mediates the octopamine-induced increase in sensitivity in spider VS-3 mechanosensory neurons. Eur. J. Neurosci., 33, 1186-1196.

Wegener, G. (1996) Flying insects: model systems in exercise physiology. Experientia, 52, 404-412. 